\title{
Children's eating behaviours and attitudes towards food in a deprived area of Belfast - a school-based survey
}

\author{
A. J. McGrath ${ }^{1}$ J. V. Woodside ${ }^{1}$ and J. Wydenbach ${ }^{2}$ \\ ${ }^{1}$ Nutrition and Metabolism Research Group, Centre for Public Health, Queen's University Belfast, Belfast, UK, BT12 6BJ \\ and ${ }^{2}$ Chartered Institute of Environmental Health Northern Ireland, York Street, Belfast, BT15 1AB, UK
}

Childhood obesity rates are increasing ${ }^{(1,2)}$. Children living in food poverty may be eating unhealthy diets, unable to afford a nutritious diet because high fat, salt and sugary foods tend to be less expensive than healthy foods e.g. fruit and vegetables ${ }^{(3,4)}$. Research also suggests that traditional eating patterns are declining with an increase in convenience, fast foods and increased snacking, particularly among young people ${ }^{(5,6)}$. This study, conducted in Northern Ireland, an area with high levels of deprivation, aimed to provide an overview of children's attitudes towards healthy food, fast food (FF), and measure their eating and purchasing behaviours in both the school term and summer holiday.

An extensive school-based survey questionnaire was conducted with children aged 11 to 17 ( $n$ 654) from post-primary schools $(n 3)$ in the top five deprived wards of Belfast. Schools were recruited by contacting the Belfast Education and Library Board to request permission and headmaster's consent from each school was gained. The questionnaire focused on usual food activity during the school term and summer holiday including breakfast, lunch, way to and home from school and views on healthy and FF.

Results confirmed that the majority of children consume snack foods, particularly fizzy drinks $(44.2 \%, 47.4 \%)$, crisps $(35.6 \%, 35.9 \%)$, chocolate $(27.7 \%, 27.2 \%)$ and sweets $(24.3 \%, 30.3 \%)$ on the way to and from school respectively. Results showed low consumption of wholegrain cereals at breakfast (6\% school, $2.6 \%$ summer). Consumption of FF and unhealthy snacks also increased in the summer. Over one fifth of children purchased food from FF outlets every day $(21.1 \%)$ and $32.7 \%$ purchased food from FF outlets one to two times per week during the summer. Furthermore, breakfast fried food consumption increased from $5.4 \%$ during school term to $20.2 \%$ in summer holiday. Afternoon purchasing activity increased for every food category during summer holiday (percentages given for school and summer holiday respectively) with fizzy drinks $(47.4 \%, 65.9 \%)$, chips $(4.9 \%, 33.8 \%)$, chocolate $(27.2 \%, 52.1 \%)$, sweets $(30.3 \%$, $49.5 \%)$ and crisps $(35.9 \%, 53.1 \%)$ being popular. However, children do have an understanding of healthy food. Fruit and vegetables $(72.8 \%)$ or salad $(63.9 \%)$ were the most common responses for what constituted a healthy food. FF was enjoyed more than healthy food; the modal rating for FF was $10(37.0 \%)$ and $5(17.6 \%)$ for healthy food on a scale of 1 to $10(1=$ least liked and $10=$ most liked).

The lack of a comparison study in an affluent area of Belfast would have determined whether eating behaviours or attitudes towards food differ between deprived and affluent areas. However, this survey highlights a number of possible areas where further research, but also education and health promotion policies could be targeted.

1. World Health Organisation (2010) Childhood overweight and obesity http://www.who.int/dietphysicalactivity/childhood/en/ [Accessed online: $14^{\text {th }}$ November 2010].

2. Central Survey Unit (2007). Northern Ireland Health and Social Wellbeing Survey 2005/06. Belfast: Central Survey Unit.

3. Institute of Public Health in Ireland (2007) "Food poverty and health" http://www.publichealth.ie/iphwork/policyandprogrammedevelopmentandevaluation/ healthinequalities/foodpoverty [Accessed online: 14th November 2010].

4. Macintyre, S (2007) Int J Behav Nutr Phys Act 4(32), 10.1186/1479-5868-4-32.

5. Kerr MA, Rennie KL, McCaffrey TA et al. (2009) Br J Nutr 101, 122-131.

6. Health Promotion Agency (2001) Eating for Health? A survey of eating habits among children and young people in Northern Ireland. Northern Ireland; Health Promotion Agency. 